BMC

Genomics

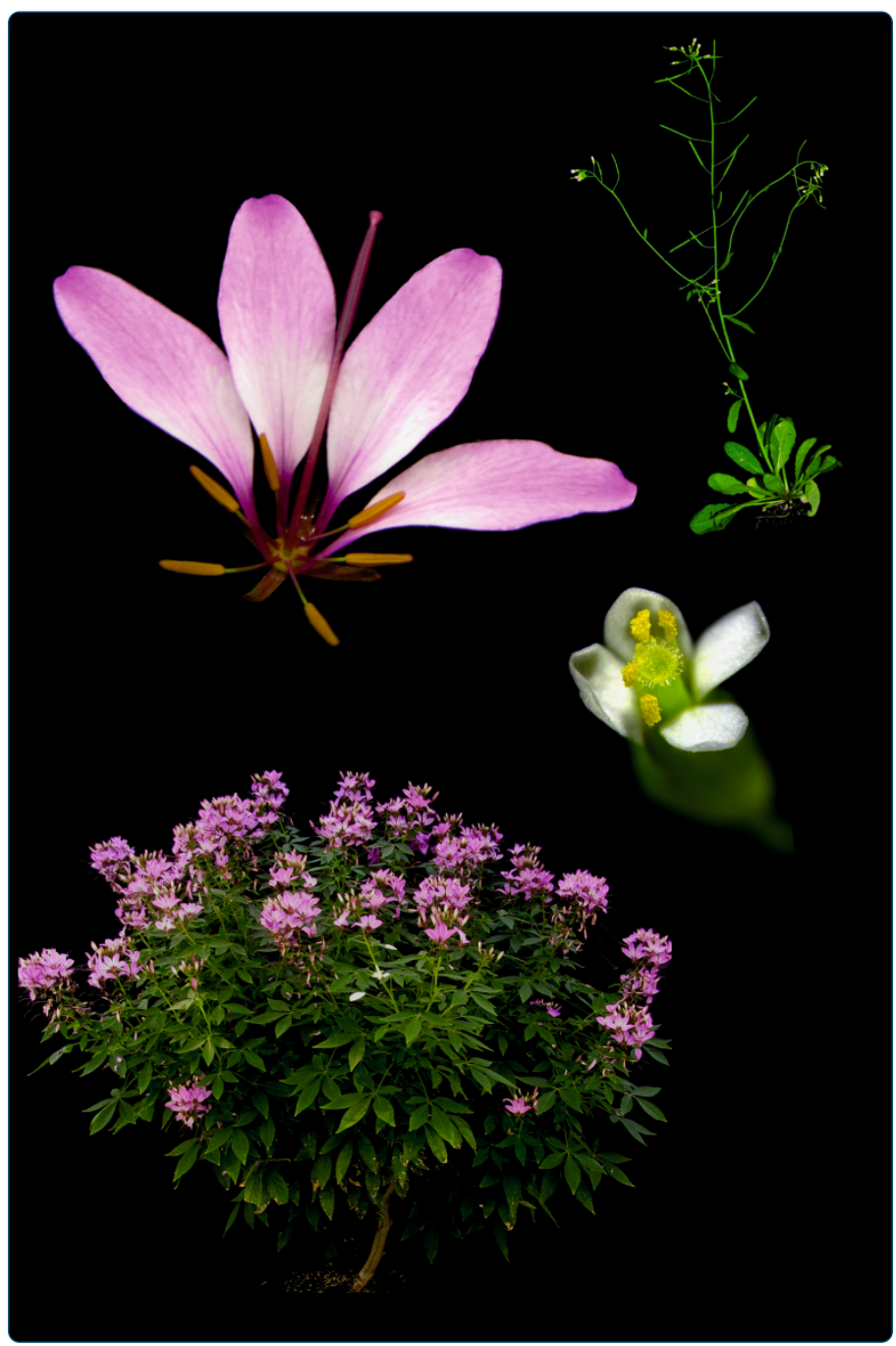

Analysis of the floral transcriptome of Tarenaya hassleriana (Cleomaceae), a member of the sister group to the Brassicaceae: towards understanding the base of morphological diversity in Brassicales

Bhide et al. 


\title{
Analysis of the floral transcriptome of Tarenaya hassleriana (Cleomaceae), a member of the sister group to the Brassicaceae: towards understanding the base of morphological diversity in Brassicales
}

\author{
Amey Bhide ${ }^{1}$, Simon Schliesky², Marlis Reich ${ }^{3}$, Andreas PM Weber ${ }^{2}$ and Annette Becker ${ }^{1 *}$
}

\begin{abstract}
Background: Arabidopsis thaliana, a member of the Brassicaceae family is the dominant genetic model plant. However, while the flowers within the Brassicaceae members are rather uniform, mainly radially symmetrical, mostly white with fixed organ numbers, species within the Cleomaceae, the sister family to the Brassicaceae show a more variable floral morphology. We were interested in understanding the molecular basis for these morphological differences. To this end, the floral transcriptome of a hybrid Tarenaya hassleriana, a Cleomaceae with monosymmetric, bright purple flowers was sequenced, annotated and analyzed in respect to floral regulators.

Results: We obtained a comprehensive floral transcriptome with high depth and coverage close to saturation analyzed using rarefaction analysis a method well known in biodiversity studies. Gene expression was analyzed by calculating reads per kilobase gene model per million reads (RPKM) and for selected genes in silico expression data was corroborated by qRT-PCR analysis. Candidate transcription factors were identified based on differences in expression pattern between A. thaliana and T. hassleriana, which are likely key regulators of the T. hassleriana specific floral characters such as coloration and male sterility in the hybrid plant used. Analysis of lineage specific genes was carried out with members of the fabids and malvids.

Conclusions: The floral transcriptome of T. hassleriana provides insights into key pathways involved in the regulation of late anthocyanin biosynthesis, male fertility, flowering time and organ growth regulation which are unique traits compared the model organism A. thaliana. Analysis of lineage specific genes carried out with members of the fabids and malvids suggests an extensive gene birth rate in the lineage leading to core Brassicales while only few genes were potentially lost during core Brassicales evolution, which possibly reflects the result of the At- $\beta$ whole genome duplication. Our analysis should facilitate further analyses into the molecular mechanisms of floral morphogenesis and pigmentation and the mechanisms underlying the rather diverse floral morphologies in the Cleomaceae.
\end{abstract}

Keywords: Tarenaya hassleriana, Arabidopsis thaliana, Floral transcriptome, Cleomaceae, Brassicaceae, Brassicales, 454 sequencing, Anthocyanins, Flower development

\footnotetext{
* Correspondence: annette.becker@bot1.bio.uni-giessen.de

'Justus-Liebig-Universität Gießen, Institute of Botany, Plant Development

Group, Heinrich-Buff-Ring 38, 35392 Gießen, Germany

Full list of author information is available at the end of the article
} 


\section{Background}

Tarenaya hassleriana, formerly known as Cleome hassleriana and sometimes erroneously referred to as Cleome spinosa [1] is a quick growing herbaceous perennial, native to Brazil and adjoining South American countries. The species belongs to the section Tarenaya and the subgenus Neocleome within the Cleomaceae [2] which includes roughly 300 species distributed throughout the tropical and subtropical regions of the world $[3,4]$. The family Cleomaceae belongs to the order Brassicales and previously Cleomaceae were thought to be more closely related to Capparaeceae but recent phylogenetic studies indicate that Cleomaceae are more closely related to and a sister family to Brassicaceae [3,5]. Molecular clock analyses suggests that Cleomaceae and Brassicaceae diverged from each other around 24.2 - 49.4 Million Years Ago (MYA) [6,7].

Analysis of normalized expressed sequence tag (EST) sequences in $T$. hassleriana and comparative genome analysis in Carica papaya, both members of the Brassicales, and in Arabidopsis thaliana belonging to Brassicaceae revealed that Cleomaceae share the most ancient gamma $(\gamma)$ whole genome duplication (WGD) with both $C$. papaya and $A$. thaliana. The sister families Brassicaceae and Cleomaceae also share the more recent beta ( $\beta$ ) WGD which is lacking in C. papaya. However, the third and most recent alpha $(\alpha)$ WGD has occurred independently in Brassicaceae and Cleomaceae. The T. hassleriana $\alpha$ WGD $($ Th- $\alpha)$ is a genome triplication and occurred approximately 13.7 MYA, while the Arabidopsis thaliana $\alpha$ WGD (At- $\alpha$ ) happened around 23.3 MYA [8]. In spite of the recent Th- $\alpha$ triplication event the genome of $T$. hassleriana is only 1.9 times the size of that of $A$. thaliana [6] and around half the size of the C. papaya genome. The small genome size of $T$. hassleriana indicates rapid diploidization, and a faster subsequent gene loss when compared to A. thaliana [6].

Cleomaceae are being intensively studied as C4 type photosynthesis evolved de novo in this group of plants. While $A$. thaliana and other Brassicaceae are C3 plants, C4 photosynthesis evolved in Cleomaceae at least three times independently in Gynandropsis gynandra, Cleome oxalidea, and Cleome angustifolia. Cleome paradoxa shows a C3 - C4 intermediate anatomy and physiology thus making Cleomaceae a model system to study C3 - C4 evolution [9]. Comparative leaf transcriptome studies by RNA-Seq have been carried out in G. gynandra (C4) and T. hassleriana (C3) to elucidate and identify novel genes and gene networks responsible for the C4 anatomy [10].

T. hassleriana (Figure 1) is also called the spider flower plant due to the long stamens which appear like appendages of spiders and is a popular ornamental plant owing to its colorful and abundant flowers. Adult plants can grow about five feet tall and several feet in diameter

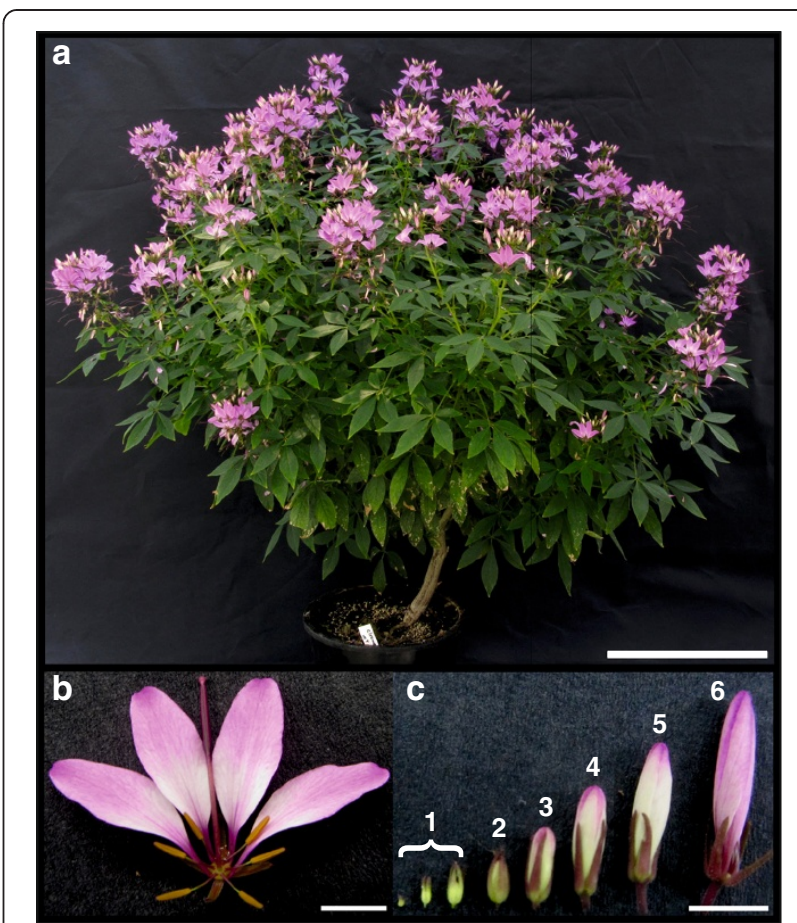

Figure 1 Tarenaya hassleriana plant. a) Morphology of a flowering T. hassleriana plant. b) Flower at anthesis showing four small purple sepals, four showy pink petals, six yellow stamens and a central gynoecium. c) Bud stages 1-6 characterized by bud length, bud stage $1(<2.5 \mathrm{~mm})$, bud stage 2 (3-4 mm), bud stage 3 (4-5 mm), bud stage $4(6-8 \mathrm{~mm})$, bud stage $5(8-10 \mathrm{~mm})$, bud stage $6(12-15 \mathrm{~mm})$. Scale bar $30 \mathrm{~cm}$ for $a$ and $5 \mathrm{~mm}$ for $b$ and $c$.

with several lateral branches. The stem and the lateral branches are soft and succulent but the main stem and older branches become woody with age. The leaves are palmate with 3-5 folioles per leaf (Figure 1a). Plants start flowering while they are in the juvenile stages and most of vegetative growth overlaps with the flowering period [11].

A typical T. hassleriana flower is zygomorphic unlike the disymmetric A. thaliana flower. Each flower has four sepals, four petals, six stamens, and a single gynoecium composed of two fused carpels (Figure 1b). The flower buds are laid out in a disymmetric bauplan during the early developmental stages which changes near anthesis and the mature flowers become zygomorphic. Conversely, in $A$. thaliana early developmental bud stages are monosymmetric and the flowers become disymmetric near anthesis [12]. C. papaya flowers on the other hand are actinomorphic at anthesis but early development has not been characterized yet. T. hassleriana inflorescences produce hermaphroditic, female, or male only flowers such that fruits are only periodically formed. The synchronous and alternate appearance of male, female, and hermaphroditic flowers in a raceme favors out-crossing, and prevents selfing except in the case of the hermaphroditic flowers [11]. This feature distinguishes $T$. hassleriana from most 
plants which are either dioecious (like C. papaya) with separate male and female plants which rarely produce hermaphroditic flowers, or monoecious like $A$. thaliana which is an obligate self-pollinated plant with hermaphroditic flowers. T. hassleriana plants are very prolific, they reseed and establish in suitable environments very easily and escape from cultivation, often becoming invasive in subtropical countries like Japan, New Zealand, parts of Australia, and the United States of America [13]. Hence many horticultural varieties, possibly like the one used in this study were developed to be sterile so that they cannot establish in non-native environments.

Also unlike most Brassicales T. hassleriana flowers are very colorful due to the presence of various anthocyanins and show 'petal fading' i.e. loss of pigmentation and dry matter associated with anthesis. This phenomenon coupled with favored cross pollination may suggest a specific role in flower - pollinator interactions or simply an age related phenomenon [14].

A close relationship to $A$. thaliana facilitates the analysis of T. hassleriana specific traits, such as flower coloration, alternating development of three types of flowers and flexible shifts from vegetative to reproductive growth, which are all not found in $A$. thaliana. Here, we describe the floral transcriptome sequence along with Transcriptome Sequencing Expression (TSE) of a horticultural T. hassleriana hybrid as a starting point for further analysis of Tarenaya flower development. Expression analysis by qRT-PCR documents the robustness of the TSE and rarefaction analysis shows that the transcriptome sequencing covers even rare transcripts. Candidate genes that may be involved in the $T$. hassleriana-specific flower developmental processes have been identified and are presented here.

\section{Methods}

\section{Plant material and growth parameters}

A T. hassleriana hybrid plant was obtained from a local garden center. It was grown in 3:1 mixture of a peat and sand based potting soil with perlite supplemented with $2 \mathrm{~g} / \mathrm{l}$ Osmocote ${ }^{\circ}$ slow release fertilizer (Scotts Deutschland $\mathrm{GmbH}$, Nordhorn, Germany). The plant was grown in a greenhouse under long day growth conditions (17 hours light and 7 hours dark) with light varying between 80 and $700 \mu \mathrm{mol} \cdot \mathrm{m}^{-2} \cdot \mathrm{s}^{-1}$ photons. Supplemental lighting of $70 \mu \mathrm{mol} . \mathrm{m}^{-2} . \mathrm{s}^{-1}$ was provided throughout the photoperiod. The temperatures in the green house varied between $20^{\circ} \mathrm{C}$ (day) and $16^{\circ} \mathrm{C}$ (night).

Tissue collection, nucleic acid extraction, and CDNA synthesis T. hassleriana floral tissue was collected for RNA and DNA extraction. The collected flower tissue was composed of equal quantity (by mass) of flowers at anthesis and each of the 6 floral bud stages defined by bud length as shown in Figure 1c).
For the floral transcriptome sequencing total RNA was extracted from the T. hassleriana floral tissue using guanidium thiocyanate-phenol-chloroform extraction protocol [15]. The polyA ${ }^{+}$mRNA was isolated using the Oligotex mRNA Minikit (Qiagen, Hilden, Germany) according to the manufacturer's instruction. The purified mRNA was analyzed for quality and quantity using the Eukaryote Total RNA Pico assay of the Agilent 2100 Bioanalyzer (Agilent Technologies, Böblingen, Germany). For the qRT-PCRs total RNA was isolated from floral tissues with the plantRNA Kit-OLS ${ }^{\circ}$ (Omni Life Science, Bremen, Germany) following the manufacturer's protocol. Genomic DNA was extracted from the floral tissue using the DNeasy ${ }^{\circ}$ Plant Mini kit (Qiagen, Hilden, Germany) according to the manual.

\section{Library preparation and $\mathbf{4 5 4}$ pyrosequencing}

200 ng of purified polyA ${ }^{+}$mRNA was used to synthesize the cDNA for sequencing with Roche Rapid Library kit (Roche) following the manual. A massively parallel pyrosequencing run was performed on a GS FLX using Titanium chemicals (Roche) with a split picotiterplate allowing two replicates to run at the same time.

\section{Assembly, annotation and gene expression}

All reads together were de novo assembled using CLC Genomics Workbench 4.9 (clcBio, Aarhus Denmark). Default parameters were chosen for the assembly. The resulting 49321 contigs were annotated against TAIR10 coding sequences (representative gene model 20110103). A reciprocal BLATX mapping was performed [16] and the best bi-directional hit per contig was kept as annotation. Chimeric contigs were determined with the pipeline provided in [17].

Gene expression was determined by mapping the reads to TAIR10 coding sequences using BLATx. The single best hit for each read was counted. Expression values were normalized to reads per Kilobase gene model per mappable million (RPKM). All reads were additionally mapped to the $T$. hassleriana floral transcriptome contigs with CLC Genomics Workbench for subsequent rarefaction analysis. The expression data for the T. hassleriana leaf transcriptome was obtained from Bräutigam et al. [10].

\section{Lineage specific gene detection}

Based on the T. hassleriana floral contigs, mappings to the transcriptomes of $A$. thaliana, B. rapa, C. papaya, and $P$. trichocarpa with an e-value cutoff of $10^{-10}$ were created in proteinspace. From those all against all mappings the 15 overlapping sets and the residual $T$. hassleriana specific set were determined using R's set methods [18]. 


\section{Quantitative reverse transcription PCR (qRT-PCR)}

For the qRT-PCRs the first strand CDNA was synthesized with the RevertAid ${ }^{\mathrm{m}} \mathrm{H}$ Minus First Strand cDNA Synthesis Kit (Fermentas, St.Leon-Rot, Germany) according to the manufacturer's protocol using an universal oligo $(\mathrm{dT})$ $\left(\mathrm{T}_{18}\right)$ primer. qRT-PCR experiments were performed according to the MIQE guidelines [19]. Exon spanning primers were then generated using PerlPrimer 1.1.21. [20]. A primer efficiency test was carried out and all the primers were tested with genomic DNA to ensure cDNA specificity. (Primer sequences are provided in Additional file 1: Table S2).

The qRT-PCR assay was performed in 96 well plates using the LightCyler 480 II (Roche, Mannheim, Germany) and analyzed with the LCS480 1.5.0.39 software. Each reaction was composed of $10 \mu \mathrm{l}$ of $2 \mathrm{x}$ DyNAmo ${ }^{\text {th }}$ Flash SYBR $^{\circ}$ Green qPCR Mastermix (Biozym Scientific GmbH, Oldendorf Germany), $2 \mu \mathrm{l}$ each of $10 \mu \mathrm{M}$ forward and reverse primers, $1 \mu \mathrm{l} \mathrm{H}_{2} \mathrm{O}$ and $5 \mu \mathrm{l}$ of diluted cDNA template. Standard dose response (SDR) curves were constructed for all the genes by using serial dilutions $(1: 50$ to $1: 50,000)$ of the cDNA template. Each reaction was performed in biological duplicates and technical triplicates along with water and RNA controls for each primer pair. The T. hassleriana ACTIN7 (ACT7) gene served as an internal control. The following PCR program was used: 7 min at $95^{\circ} \mathrm{C} ; 45$ cycles of $10 \mathrm{~s}$ at $95^{\circ} \mathrm{C}, 15 \mathrm{~s}$ at $60^{\circ} \mathrm{C}, 15 \mathrm{~s}$ at $72^{\circ} \mathrm{C}$, followed by a melting curve of $5 \mathrm{~s}$ at $95^{\circ} \mathrm{C}, 1 \mathrm{~min}$ at $65^{\circ} \mathrm{C}$ and $30 \mathrm{~s}$ at $97^{\circ} \mathrm{C}$. The Absolute Quantification analysis and the quantification cycle (Cq) were calculated according to the Fit Points method using the LCS480 1.5.0.39 software. The amplification efficiency was calculated using the SDR for each gene. The raw data were analyzed according to the relative standard curve method and the fold difference between the expression of $A C T 7$ and the genes of interest was calculated using the comparative Cq method ( $\Delta \Delta \mathrm{Cq})$ [21]. A one way ANOVA was performed to calculate the statistical significance of the difference between the three expression values.

\section{Comparison of $A$. thaliana and $T$. hassleriana floral gene expression and $\mathrm{GO}$ annotations}

In order to identify genes that may play a role in the T. hassleriana specific floral traits, transcripts specific for the $T$. hassleriana flower, not expressed in the A. thaliana flower and vice versa were identified. Microarray expression data [22] for A. thaliana flower stages 1-6, 9, 10-11, 12, 15 (ATGE_29_A2, B2, C2; ATGE_31_A2, B2, C2; ATGE_32_A2, B2, C2; ATGE_33_A2, B2, C2; ATGE_39_A2, B2, C2) were downloaded from

The Arabidopsis Information Resource (TAIR), http:// arabidopsis.org/servlets $/$ TairObject?type $=$ hyb_descr collection\&id=1006710873\#497, on, Nov 202012.

Of the 22,746 microarray probes hybridizing to 23,570 genes, only 21,107 probes hybridizing to unique transcripts were considered for the analysis. A dataset corresponding to the expression of these 21,107 transcripts in the A. thaliana floral transcriptome was compiled. Expression of a gene in at least one floral stage and sample subset was considered as presence of the transcript in the A. thaliana floral transcriptome. The presence or absence of homologous transcripts in the T. hassleriana floral transcriptome was analyzed. A list of putative T. hassleriana orthologs of $A$. thaliana genes expressed in $T$. hassleriana floral transcriptome but not in the A. thaliana floral transcriptome was constructed. Also, transcripts present in the A. thaliana flower transcriptome but homologs absent in the T. hassleriana transcriptome were identified. Gene Ontology (GO) annotations were assigned to genes expressed exclusively in the A. thaliana or T. hassleriana transcriptome using the online tool for functional annotation Blast $2 \mathrm{GO}^{\circ}$ [23] by performing a BLASTX with a cutoff value of $1 \mathrm{e}^{-100}$ as this value showed robust matches of GO annotations to TAIR annotations.

GO annotations were assigned to $T$. hassleriana lineage specific sequences, and other sequences shared by Cleomaceae with the Brassicaceae, Brassicales or lost in the Brassicaceae using Blast2GO ${ }^{\circ}$ [23] by performing BLASTX and BLASTN with cutoff values of $1 \mathrm{e}^{-10}$.

\section{Rarefaction analysis}

Rarefaction analysis is commonly used in ecological research defining species richness as a function of sequencing effort. Such an analysis can be broadened to genomics as long as the data are distributed as described in the original paper defining the underlying equation [24]. Hale et al. [25] already calculated rarefaction curves for transcriptome analysis of a polyploid lake sturgeon. Here, we applied rarefaction analysis to ascertain whether sequencing depth and coverage was sufficient to draw a comprehensive picture of the transcriptome of Cleome. Thus, three different libraries were created: one data set for each biological replica as well as a merged one. Data sets were constructed by listing each gene (defined by a contig) with its read support. Rarefaction curves were calculated using the program aRarefactWin (https://www.uga.edu/strata/software/). Hereby, genes were randomly resampled and it was recorded which gene of the library was identified with which frequency. This procedure was repeated 1,000 times. Then, the average number of each gene found was plotted for different read numbers drawing a curve whose slope indicated if sequencing effort was deep enough. This was the case when the curve flattened and ran into a plateau.

\section{Results}

\section{Sequencing}

Massively parallel pyrosequencing of two samples of T. hassleriana (Additional file 1: Table S1) yielded 1,254,286 sequencing reads in total. The sequencing raw data are 
deposited in the DDBJ (DNA Data Bank Japan, http:// trace.ddbj.nig.ac.jp/index_e.html) under the experiments SRR1051360 and SRX393170 https://trace.ddbj.nig.ac.jp/ DRASearch/run?acc=SRR1051360 and (https://trace. ddbj.nig.ac.jp/DRASearch/experiment?acc=SRX393170) The histogram of reads by length (Additional file 2: Figure S1a) showed an average read length of $\sim 316$ nucleotides. Roughly $45 \%$ of the reads could be mapped against A. thaliana TAIR10 coding sequences for counting gene expression.

Assembling the reads de novo resulted in 49,237 contigs with an N50 of 690 bases (Additional file 2: Figure S1b). Of these, 41,320 could be annotated by mapping against Arabidopsis. 1.1\% (537) chimeric contigs could be detected in the assembly.

\section{Rarefaction analysis}

Rarefied libraries were constructed separately for the two biological replicates 1 and 2 and a merged sample to illustrate possible differences in gene discovery rates. Although the gene discovery rate of replicate 1 was less than the one of replicate 2 (Figure 2), the curves for both replicates indicated that a larger part of the $T$. hassleriana floral transcriptome was detected as the curves already flattened. However, the merging of the information of both libraries affected the overall output as the rarefaction curve reached nearly a plateau (Figure 2). This shows that each library comprised genes not detected with the other one. Thus, the merged data set allows drawing a detailed view of the transcriptome of $T$. hassleriana. Increasing the sequencing depth would only result in the detection of extremely rare genes.

\section{qRT-PCR expression analysis validates transcriptome sequencing expression (TSE)}

The robustness of expression data generated by the transcriptome sequencing was analyzed independently

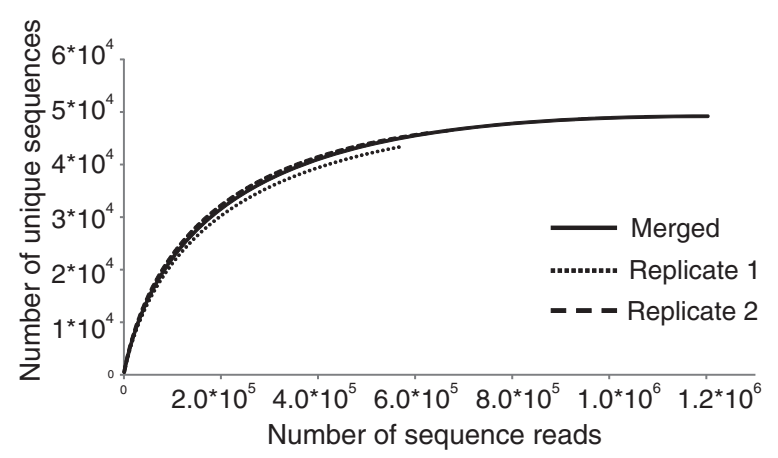

Figure 2 Rarefaction for transcriptome depth and coverage analysis. Rarefaction curves for both libraries representing expected gene coverage rates. Curves were constructed for the two biological replicates 1 and 2 and a merged sample. using a qRT-PCR assay (Figure 3). A normalized expression profile for $T$. hassleriana reads mapped to A. thaliana CDS sequences was created by calculating the ratio of reads mapped to an individual gene against the reads mapped to $A$. thaliana ACT7. A subset of 14 genes was randomly chosen to represent genes with high (normalized expression ratio 1.0 - 10.0, Figure 4a), moderate (normalized expression ratio $0.3-1.0$, Figure $4 \mathrm{~b}$ ) and low (normalized expression ratio $0.05-0.3$, Figure 4c) expression levels. The expression of the putative $T$. hassleriana orthologs of the A. thaliana genes RBCS1A, MVP1, GAPC1, TT4, BGLUC19, GAMMAVPE, ATP3, SCE1A, SFGH, ARF6, PGLUHYD, GI, OMR1, and SPL7 was analyzed in $T$. hassleriana floral tissue (A. thaliana gene identifier, full gene names are shown in Additional file 1: Table S3). The qRT-PCR expression data were also normalized to the expression of the T. hassleriana ACT7.

Generally we found a better match of transcript abundance detected by qRT-PCR in T. hassleriana as compared to reads mapped to the $A$. thaliana orthologs (TSE1) than to the T. hassleriana contigs (TSE2). A correlation plot for the comparison of expression measured by qRT-PCR and TSE was generated (Additional file 2: Figure S3). When all the 14 gene expressions by the two methods were plotted a positive linear correlation was observed (Additional file 2: Figure S3a) as indicated by a $\mathrm{R}^{2}$ value 0.55 . The expression of MVP1 and BGLUC19 gene homologs which belong to big gene families with 41 and 66 homologs in $A$. thaliana respectively was the most significant outlier in this plot. When the expression data for the MVP1 and BGLUC19 gene homologs were removed and the data plotted again a very strong positive linear correlation between TSE1 and qRT-PCR expression values was obtained with an $\mathrm{R}^{2}$ value 0.91 (Additional file 2: Figure S3b). This indicated that TSE1 approach for measuring gene expression was very robust except for genes belonging to large gene families with highly similar homologs in which case the read mapping may be incorrect. Nonetheless a positive linear expression correlation for all genes corroborates the TSE1 expression data. In particular, similar normalized fold expression between qRT-PCR data and reads mapped to the $A$. thaliana orthologs were observed in the genes RBCS1A (high expression), ATP3, SCE1A, SFGH (moderate expression), and ARF6, PGLUHYD, OMR1, and SPL7 (low expression) P >0,01 (Additional file 1: Table S4 shows the comparative $\mathrm{P}$ values for the ANOVA tests). In case of $T$. hassleriana homologs of genes GAMMAVPE, MVP1 and TT4 the transcript abundance detected by qRT-PCR was more similar when reads were mapped to the T. hassleriana contigs (TSE2) P $>0,01$. In case of the GAPC1 and BGLUC19 homologs the difference between qRT-PCR expression and TSE1 and TSE2 was statistically significant $\mathrm{P}<0,01$. It was further observed that the number of reads mapped to the $T$. hassleriana contigs was in all 
Figure 3 Comparative analysis of Transcriptome Sequencing based Expression data (TSE) with qRT-PCR expression data. The TSE and the GRT-PCR expression were normalized with the putative T. hassleriana ACTIN7 homolog. The first column represents the TSE 1 where the $T$. hassleriana reads are mapped on to the $A$. thaliana CDS sequences; the second column represents the TSE 2 where the $T$. hassleriana reads are mapped on the $T$. hassleriana contigs and the third column represents the GRT-PCR expression data. a: Comparison of TSE and qRT-PCR in genes with high expression (750-2000 RPKM, b: Comparison of TSE and GRT-PCR in genes with moderate expression (150-300 RPKM) and c: Comparison of TSE and qRT-PCR in genes with low gene expression (25-150 RPKM). The error bars represent the standard deviation and the P-values for statistical significance between expression values are presented in Additional file 1: Table S4. b

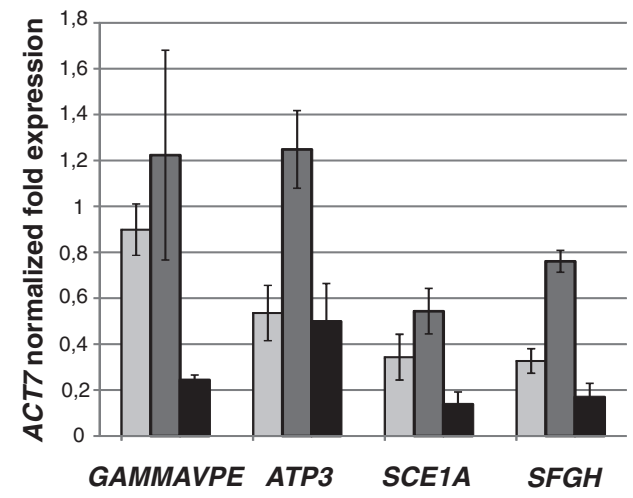

Putative $T$. hassleriana orthologs of $A$. thaliana genes

C

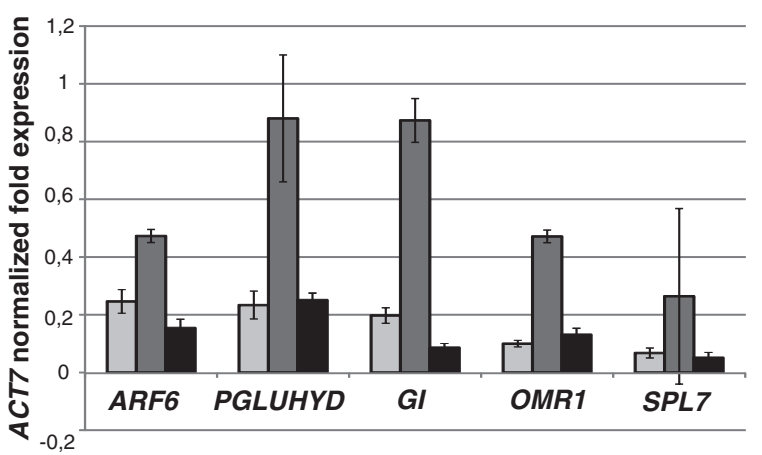

Putative $T$. hassleriana orthologs of $A$. thaliana genes

TSE $1 T$. hassleriana reads mapped to A. thaliana CDS

TSE 2 T. hassleriana reads mapped to T. hassleriana contigs

T. hassleriana q RT-PCR expression cases, with the exception of TT4, grossly overestimating gene expression.

\section{Expression of genes controlling floral traits in the flower} and leaf transcriptome

Genes controlling various floral traits and flower development in A. thaliana, Antirrhinum majus, Fagopyrum esculentum etc. were identified based on literature [26-31]. The expression pattern of their putative $T$. hassleriana orthologs identified by a bidirectional BLATX search with the $A$. thaliana CDS sequences was analyzed in the flower and leaf transcriptomes to learn more about the regulation of the special floral traits of $T$. hassleriana (Figure 4). The selected genes were first grouped into different classes such as homeotic transcription factors, regulators of homeotic genes etc. and ordered within their groups according to transcript abundance. Of the genes analyzed, 49 (41.9\%) were specific to the flower transcriptome and not found in the leaf transcriptome. (A. thaliana gene identifier, full gene names are shown in Additional file 1: Table S3).

Amongst the putative class $\mathrm{ABCDE}$ homeotic transcription factor orthologs, the highest expression was observed among the class $\mathrm{B}$ gene homologs $A P 3$ and $P I$ and the class E gene homologs SEP1 and SEP3. The putative ortholog of the $C$ class gene $A G$ was expressed at a 10 fold lower magnitude compared to the class $\mathrm{B}$ and $\mathrm{E}$ genes. The expression of the putative orthologs of the D class genes SHP1, SHP2 and STK the expression of which regulates the ovule and fruit development in A. thaliana was found to be considerably lower, when compared to the class $\mathrm{ABCE}$ genes. $A P 3, S E P 3, S E P 1$, and $S T K$ transcripts were not present in the leaf transcriptome while $P I, A P 1, A P 2$, and SEP4 are expressed at a very low level in leaves. In addition to these, 25 other putative MADS box transcription factors without floral homeotic function that are members of the MIKC, $M \alpha, M \beta, M \gamma, M \delta$ subfamilies were also found to be expressed in the floral transcriptome.

Amongst the genes putatively regulating the class ABCDE homeotic transcription factors, the LUG, LUH 


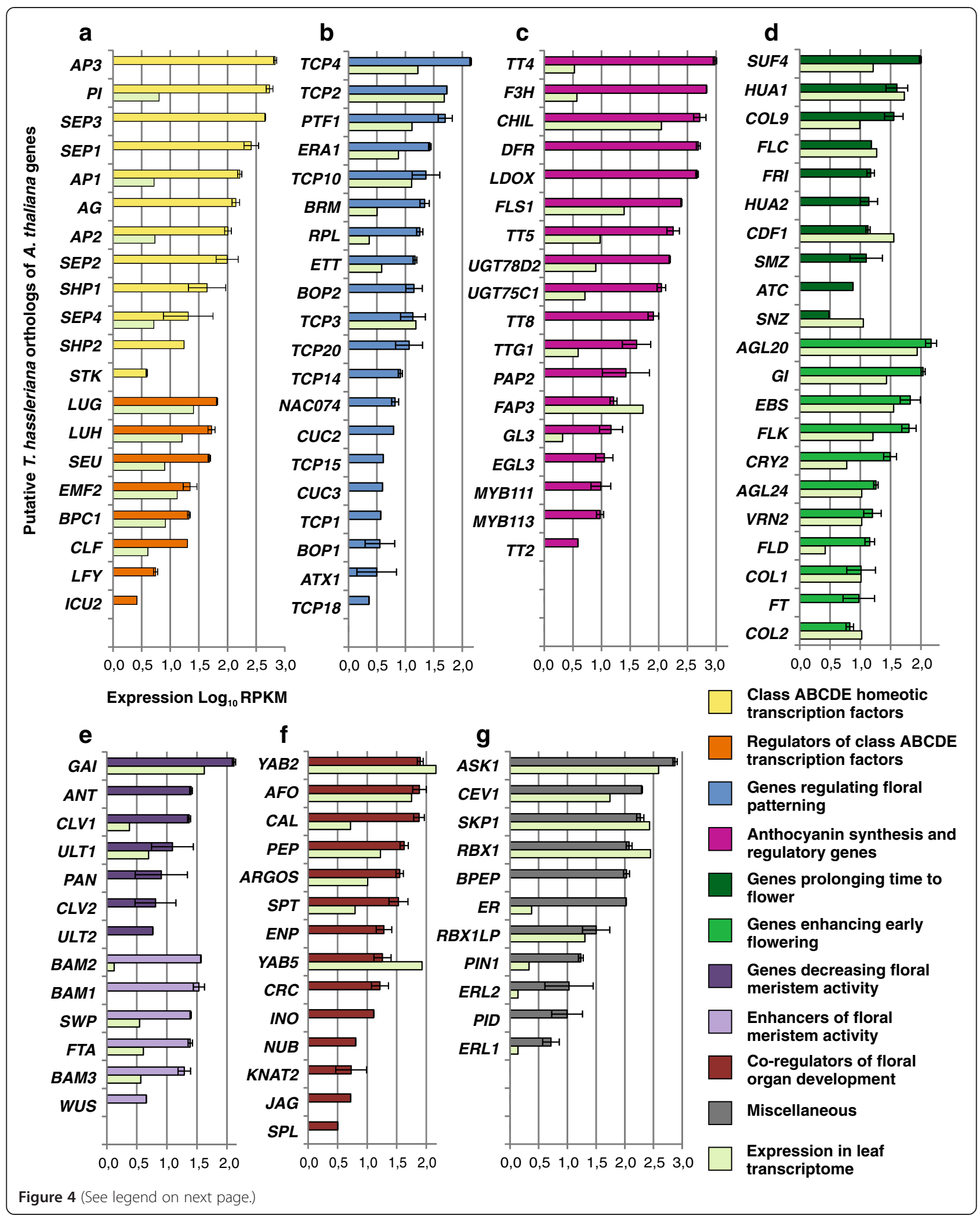


(See figure on previous page.)

Figure 4 Expression of $T$. hassleriana orthologs of $A$. thaliana genes regulating various floral characteristics in the $T$. hassleriana flower and leaf transcriptomes. The putative orthologs are plotted on the $Y$ axis and the Transcriptome Sequencing Expression (TSE 1) which is the $\log _{(10)}$ of RPKM is plotted on the X axis. The error bars show standard deviation; a: Expression of class ABCDE homeotic transcription factors and their regulators in the floral and leaf transcriptomes of T. hassleriana, $\mathbf{b}$ : Genes regulating patterning and symmetry, $\mathbf{c}$ : Genes involved in synthesis and regulation of anthocyanins, $\mathbf{d}$ : Genes regulating time to flower, e: Positive and negative regulators of floral meristem activity, f: Co-regulators of floral organ development, g: Miscellaneous group genes involved in flower development.

and SEU orthologs showed highest expression in the flower transcriptome, while a 10 fold lower expression of these genes was observed in the leaf transcriptome. The expression of the $L F Y$ homolog was also observed in the floral transcriptome albeit at very low levels. Interestingly, putative homologs of genes regulating class B gene activities like UFO and SUP and class A gene activity like $S A P$ were not identified in the floral transcriptome library. The orthologs for genes regulating patterning and symmetry also showed expression in the floral transcriptome. The putative orthologs of TCP4, TCP2, and PTF1 showed the highest expression. The expression of these genes was also observed in the leaf transcriptome; in case of the TCP4 ortholog a 100 fold higher expression was observed in the floral transcriptome when compared to the leaf transcriptome, while the TCP2 ortholog expression was almost equal in both the transcriptomes. Comparatively low expression of other putative patterning gene orthologs like TCP14, TCP15, TCP18, CUC2 and CUC3 was also observed specific to the floral transcriptome.

While the $A$. thaliana flowers are mostly free of pigments, the petals and reproductive organs of T. hassleriana are pink and dark magenta and hence the expression of putative orthologs of genes regulating anthocyanin production, regulation, and deposition was analyzed. Very high expression was observed for the putative orthologs of TT4, F3H, CHIL, DFR and LDOX. Most genes show a higher expression in flowers than in leaves and for several, such as DFR, $L D O X$, and TT8, expression is specific to the flower suggesting key roles in flower pigmentation. Very low expression of TT4 and F3H orthologs (about 300 and 200 fold lower respectively) was observed also in the leaf transcriptome, whereas the CHIL ortholog expression was only about 5 fold lower in the leaf transcriptome. The spatiotemporal expression pattern of $A$. thaliana orthologs of these genes was investigated in A. thaliana using the Arabidopsis eFP Browser (http:// bar.utoronto.ca/efp/cgi-bin/efpWeb.cgi) [32]. The expression patterns for the homologs of TT4, F3H, CHIL, and FLS1 was very similar in T. hassleriana and A. thaliana. The enzymes encoded by these genes are required for the synthesis of flavonoids like quercitin, dihydroquercitin, myricetin etc., which are intermediates of anthocyanin biosynthesis. The products of the genes DFR, LDOX, UGTD2 which were found to be expressed in in the T. hassleriana floral transcriptome but only in senescing leaves in A. thaliana (Table 1) are involved in downstream processes that catalyze the conversion of the flavonoids into anthocyanins like Pelargonidin and Cyanidin which determine the characteristic pink-magenta flower color. Genes like $P A P 2$, $M Y B 111, M Y B 113$, and EGL3 are regulators of flavonoid and anthocyanin biosynthesis and were also expressed in $T$. hassleriana floral tissue whereas in A. thaliana their expression was restricted to senescing leaves and seeds during early stages of embryo development.

Expression of gene orthologs governing time to flower was also analyzed. Expression of both antagonistic groups of genes that prolong time to flower or enhance the transition into flowering was observed. Among the orthologs inducing flowering AGL20, GI, EBS, and FLK had the highest expression; expression of these genes was also observed in the leaf transcriptome at very comparable levels. Amongst the orthologs of genes delaying flowering SUF4, HUA1, COL9, and FLC had high levels of expression which was also observed at comparable levels in the leaf transcriptome. The orthologs of FRI, HUA2, SMZ, and ATC showed moderate to low floral transcriptome specific expression.

$T$. hassleriana homologs of meristem activity regulators, such as GAI, ANT and CLV1 which are involved in decreasing meristem proliferation was observed at high levels in the flower and varying levels in the leaf transcriptome while $A N T$ expression was not detected in the leaf transcriptome. Putative homologs of genes BAM1,BAM2, BAM3 and WUS which enhance meristem proliferation were also found to have moderate expression levels in the floral transcriptome. Interestingly, putative homologs for FTA, ERA1, and STM, were found to be expressed in the floral transcriptome as their $A$. thaliana counterparts show very low expression the flower.

Another important category of gene orthologs analyzed for expression are the genes that co-regulate floral organ development alongside the ABCDE floral homeotic transcription factors. High expression was observed in case of orthologs of $Y A B 2, A F O$ and PEP in both the floral and leaf transcriptomes whereas the expression of the $C A L$ ortholog was about 100 fold higher in the flower transcriptome. Other floral organ developmental regulators, such as ENP, CRC, INO, NUB, JAG, and SPL were not identified in the $T$. hassleriana leaf transcriptome, but only in floral transcriptome whereas they are also expressed in $A$. thaliana leaves at very low levels. 
Table 1 Genes putatively involved in anthocyanin synthesis, regulation, and deposition found in the floral transcriptome of $T$. hassleriana and the expression of their putative orthologs in $A$. thaliana tissues and developmental stages

\begin{tabular}{|c|c|}
\hline $\begin{array}{l}\text { Gene homologs } \\
\text { expressed in } \\
T \text {. hassleriana floral } \\
\text { transcriptome }\end{array}$ & Expression in A. thaliana \\
\hline TT4 & Buds, senescent leaf, seed (globular embryo stage) \\
\hline $\mathrm{F} 3 \mathrm{H}$ & $\begin{array}{l}\text { Buds, petal, seed (globular and torpedo stage } \\
\text { embryo) }\end{array}$ \\
\hline CHIL & $\begin{array}{l}\text { Buds, petal, young silique, seeds (globular and } \\
\text { torpedo stage embryo) }\end{array}$ \\
\hline$D F R$ & $\begin{array}{l}\text { Senescent leaf, young silique, seed (heart stage } \\
\text { embryo) }\end{array}$ \\
\hline LDOX & $\begin{array}{l}\text { Senescent leaf, young silique, seed (heart stage } \\
\text { embryo) }\end{array}$ \\
\hline FLS1 & $\begin{array}{l}\text { Buds, petal, seeds (torpedo and walking stick } \\
\text { stage embryo) }\end{array}$ \\
\hline TT5 & $\begin{array}{l}\text { Buds, petal, carpel, seed (globular and heart stage } \\
\text { embryo) }\end{array}$ \\
\hline UGTD2 & $\begin{array}{l}\text { Senescent leaf, seed (curled cotyledon, green } \\
\text { cotyledon stage embryo) }\end{array}$ \\
\hline UGTC1 & Senescent leaf \\
\hline TT8 & $\begin{array}{l}\text { Young siliques, seeds (heart, walking stick stage } \\
\text { embryo) }\end{array}$ \\
\hline TTG1 & $\begin{array}{l}\text { All plant organs, high expression in cauline and } \\
\text { senescent leaves, young siliques, seeds (heart and } \\
\text { torpedo stage embryo) }\end{array}$ \\
\hline PAP2 & Senescent leaf \\
\hline FAP3 & $\begin{array}{l}\text { Cauline leaf, young siliques, seeds (Heart, torpedo, } \\
\text { walking stage embryo) }\end{array}$ \\
\hline GL3 & Expression data not available \\
\hline EGL3 & $\begin{array}{l}\text { Shoot apex (vegetative, floral transition, } \\
\text { inflorescence), young silique, seeds (globular, } \\
\text { torpedo, walking stick stage embryo) }\end{array}$ \\
\hline MYB111 & Petals, shoot apex (inflorescence) \\
\hline MYB113 & $\begin{array}{l}\text { All plant organs, high expression in pollen, seeds } \\
\text { (curled cotyledon and green cotyledon embryo } \\
\text { stage) }\end{array}$ \\
\hline TT2 & $\begin{array}{l}\text { Young siliques, seeds (globular and heart stage } \\
\text { embryo) }\end{array}$ \\
\hline
\end{tabular}

No expression was observed for $R O X Y$ gene homologs which are responsible for anther and male gametophyte development downstream of $S P L$.

Other putative homologs of $A$. thaliana floral regulators were identified amongst them were the highly expressed homologs of genes ASK1, CEV1, SKP1, RBX1, which are part of SCF ubiquitin protein ligase complexes which regulate multiple aspects of flower development together with UFO in A. thaliana [33]. Homologs of genes ER, ERL1 and $E R L 2$ which are protein kinases that influence meristem cell fate and patterning in the inflorescence meristem were also highly expressed. Interestingly the homolog of BPEP was found to be expressed only in the floral transcriptome, while the two distinct BPEP transcripts in A. thaliana are expressed in the floral as well as in vegetative organs respectively. Homologs of genes PIN1 and PID were also expressed which are known to affect size, floral organ number and total number of flowers in A. thaliana.

This in silico expression analysis of genes related to flower development demonstrates that with the chosen RNAseq method we are able to monitor gene expression in logarithmic scales covering more than two magnitudes. In addition, the two library preparations for this sequencing experiment show only rarely any difference in RPKM. Detailed expression analysis of putative $T$. hassleriana homologs of $A$. thaliana genes in the $T$. hassleriana floral transcriptome is provided in Additional file 3 along with the AGI identifiers.

\section{Characterization of genes putatively governing sterility in T. hassleriana}

The particular T. hassleriana hybrid used in this study was sterile. While orthologs of $A$. thaliana regulators of anther development were expressed in the T. hassleriana flower, no expression of ROXY1 and ROXY2 was detected. These two genes redundantly control the anther lobe and pollen mother cell differentiation downstream of SPL [34]. The genome of one of the parents of this hybrid, T. hassleriana Purple Queen (ES1100) was recently published [35] and this plant, unlike its hybrid offspring is fertile. Only ROXY1 ortholog was found in the T. hassleriana genome To learn more about the possible causes for the sterility we compared the expression pattern of homologs of SPL, ROXY1 and their A. thaliana downstream targets $D Y T 1$ and MYB35 affecting stamen development and microsporogenesis in these two plants by qRT-PCR at small, medium and large buds (Figure 5).

Expression analysis by qRT-PCR indeed revealed that the expression of the ROXY1 homolog was very low ( $10^{3}$ fold lower compared to ACT7) and well beyond the scope of detection by RNA seq. ROXY1 expression was down regulated in the sterile hybrid only at bud stage $\mathrm{M}$ when compared to the fertile parent the (Figure $5 \mathrm{~b}$ ) whereas it was similar to the parent at the younger and later developmental stages. Along with the down regulation of ROXY1, expression for the DYT1 and MYB35 homologs which most likely act downstream of $R O X Y 1$ was also down regulated in stage $M$ buds. In stages other than $\mathrm{M}$, the expression of DYT1 and MYB35 homologs in the sterile $T$. hassleriana hybrid was several fold higher than the respective expression in fertile parent buds in both the early and late developmental stages. Expression of the SPL homolog in the sterile hybrid buds was 3-4 fold higher than the fertile plant buds in stages $S$ and $M$ whereas in stage latter $L$ the expression was 8 fold. Thus our expression data suggest that the complex network governing 


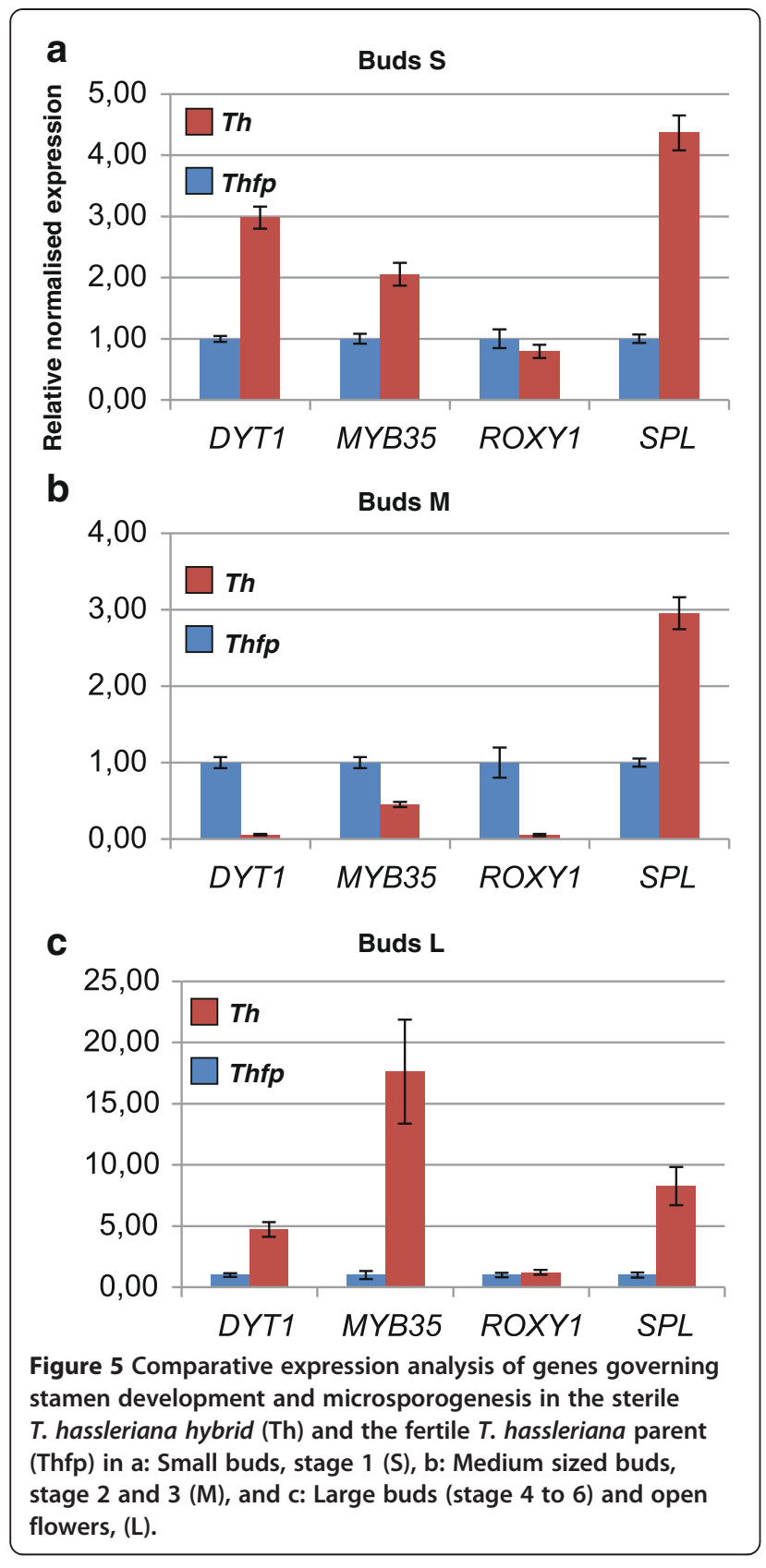

stamen development and microsporogenesis is disrupted in the T. hassleriana hybrid which could provide a causal link to its sterility.

\section{Characterization of $T$. hassleriana floral transcriptome specific genes in comparison to $A$. thaliana}

We described above that the flower of T. hassleriana is morphologically distinct from the A. thaliana flower and our aim was to identify genes that may contribute to the differences by comparing the $A$. thaliana floral transcriptome with that of $T$. hassleriana. However, as our data are based on RPKM and the A. thaliana are microarray data the two datasets may be compared only qualitatively but not quantitatively. We thus chose the more careful approach to score only for presence/absence of transcripts of $A$. thaliana/T. hassleriana putatively orthologous gene pairs. Of the 21,107 genes in A. thaliana for which microarray expression data for the floral transcriptome could be compiled, 1200 genes were not expressed in the A. thaliana. The expression analysis of these gene homologs in the T. hassleriana revealed that a majority of these genes $(\sim 750)$ were also not expressed in the T. hassleriana floral transcriptome. But 351 gene homologs were identified that were expressed differentially amongst the floral transcriptomes of the two species. These differentially expressed Tarenaya transcripts were assigned $\mathrm{GO}$ annotations using Blast2 $\mathrm{GO}^{\circ}$ by performing a BLASTX search with a cut off value of $\mathrm{e}^{-100}$ to identify the molecular processes that are distinct between $T$. hassleriana and A. thaliana. 81 genes were annotated as genes with unknown function. The remaining 270 genes were assigned multiple GO annotations based on the biological processes associated with the function of these genes (Additional file 1: Table S5). Of special interest were genes annotated to be involved in anthocyanin accumulation, cell growth, flower development and other developmental processes. Candidate genes were selected for further analysis (Table 2). High expression of PGP10 homolog, a gene involved in anthocyanin accumulation in response to UV light was observed in the $T$. hassleriana floral transcriptome whereas its expression is limited to pollen in A. thaliana. The homolog of TTFP which codes for a tyrosine transaminase family protein was also expressed at high levels in the T. hassleriana floral transcriptome; this gene is involved in regulation of cell growth in response to external stimulus and is primarily expressed in the roots of $A$. thaliana. Other notable gene homologs involved in various aspects of cell growth were LRX2, HAT4 and PIP5K3. Of the gene homologs involved in various aspects of floral development, prominent were ICMTA and TEM2. ICMTA is an enzyme belonging to the methyltransferase family, which is inducted during floral morphogenesis. TEM2 is a transcription factor known for its role in flowering time regulation by controlling $F T$ expression. Amongst the genes annotated as genes governing various aspects of development were JAL33, MTSP1, EMB2217 and GLUDOXRP which are involved in embryo and root development.

\section{Identification and characterization of Cleome lineage specific genes}

To identify genes shared between Cleome and other closely related rosids and genes that are specific to the Cleome lineage a BLASTX search with a cut off value of $\mathrm{e}^{-10}$ was performed with the 49,237 Tarenaya floral transcriptome contigs against the A. thaliana, Brassica rapa, C. papaya 
Table 2 Selection of homologous gene pairs in which the homologs of T. hassleriana are expressed in the flower and the $A$. thaliana homolog expression is absent from the flower

\begin{tabular}{|c|c|c|c|c|}
\hline Gene abbreviation & Process/protein family & GO ID & $\begin{array}{l}\text { T. hassleriana } \\
\text { TSE (RPKM) }\end{array}$ & Expression in A. thaliana \\
\hline \multicolumn{5}{|c|}{ Anthocyanin accumulation } \\
\hline PGP10 & multidrug pheromone mdr abc transporter family & GO:0043481 & 63.79 & Mature pollen \\
\hline \multicolumn{5}{|l|}{ Cell growth } \\
\hline TTFP & tyrosine transaminase family protein & GO:0001560 & 194.20 & Root \\
\hline$A R P 2$ & actin-related protein 2-like & GO:0009825 & 28.21 & $\begin{array}{l}\text { Senescent leaf, cauline leaf, buds, flower, } \\
\text { inflorescence shoot apex }\end{array}$ \\
\hline$H B-2$ & homeodomain-leucine zipper protein & GO:0009826 & 28.07 & $\begin{array}{l}\text { Young leaf, mature leaf, cauline leaf, senescent } \\
\text { leaf, pedicel, seed (torpedo stage embryo) }\end{array}$ \\
\hline$\angle R X 2$ & leucine-rich repeat extensin-like protein 1 & GO:0009826 & 22.97 & $\begin{array}{l}\text { Young leaf, pollen, seed } \\
\text { (cotyledon stage embryo) }\end{array}$ \\
\hline PLLSP & pectate lyase family protein & GO:0042547 & 12.85 & $\begin{array}{l}\text { Young leaf petiole, mature leaf (distal end), } \\
\text { seed (curled cotyledon stage embryo) }\end{array}$ \\
\hline \multicolumn{5}{|l|}{ Flower development } \\
\hline ICMTA & protein-s-isoprenylcysteine o-methyltransferase a & GO:0009908 & 37,04 & $\begin{array}{l}\text { Young leaf, cauline leaf, senescent leaf, young } \\
\text { silique, seed (heart and torpedo stage embryo) }\end{array}$ \\
\hline SBP3 & selenium-binding protein & GO:0048573 & 24,26 & Imbibed seed \\
\hline BTB/POZ P & BTB/POZ domain-containing protein & GO:0048439 & 12,55 & Petals stamens \\
\hline TEM2 & $\begin{array}{l}\text { ap2 erf and b3 domain-containing } \\
\text { transcription factor rav2 }\end{array}$ & GO:0009910 & 12,28 & Cotyledon, young leaf, senescent leaf \\
\hline \multicolumn{5}{|l|}{ Development } \\
\hline JAL33 & jacalin-like lectin domain-containing protein & GO:0009793 & 302,33 & Root, hypocotyl \\
\hline MTSP2 & caffeoyl- o-methyltransferase & GO:0048316 & 47,03 & $\begin{array}{l}\text { Seed (curled cotyledon, green cotyledon } \\
\text { embryo stage), dry seed }\end{array}$ \\
\hline MTSP1 & $\begin{array}{l}\text { s-adenosyl-I-methionine-dependent } \\
\text { methyltransferase-like protein }\end{array}$ & GO:0010089 & 40,59 & $\begin{array}{l}\text { Seed (walking stick, curled cotyledon, green } \\
\text { cotyledon embryo stage) }\end{array}$ \\
\hline LRRTPKP & $\begin{array}{l}\text { Irr receptor-like serine threonine-protein } \\
\text { kinase rch1-like }\end{array}$ & GO:0048443 & 15,26 & Root, seed (torpedo stage embryo), imbibed seed \\
\hline CYP705A27 & cytochrome p450 & GO:0048589 & 11,86 & Root, seed (cotyledon embryo stage), dry seed \\
\hline EMB2271 & $\begin{array}{l}\text { u3 small nucleolar rna-interacting } \\
\text { protein 2-like }\end{array}$ & GO:0009553 & 11,81 & Stamen \\
\hline CYP705A & cytochrome p450 & GO:0048589 & 11,54 & Root \\
\hline GLUDOXRP & glutaredoxin-related protein & GO:0048653 & 7,40 & $\begin{array}{l}\text { Pollen, seed (walking stick, curled cotyledon, } \\
\text { green cotyledon stage embryo) }\end{array}$ \\
\hline LRRRPK & receptor-like protein kinase 2 -like & GO:0048443 & 3,50 & Imbibed seed, root \\
\hline
\end{tabular}

(all malvids, order Brassicales) and Populus trichocarpa (fabid, order Malpighiales) protein databases in a systematic manner (Figure 6a). This allows the assessment of gene births and gene losses in the rosid lineage. Figure $6 \mathrm{~b}$ shows the result of the comparative analysis: A large number of the contigs 37,989 (subset I) represent the sequences shared between malvids and fabids. According to our analysis, only 684 genes are shared between all Brassicales, but 1375 genes (subset B) are shared between the core Brassicales, which include $T$. hassleriana, $A$. thaliana, and $B$. rapa [36]. This suggests a high rate of gene births in the lineage leading to core Brassicales after their split from the lineage leading to C. papaya. Conversely, 148 genes (subset K) are shared between T. hassleriana, C. papaya and P. trichocarpa and not found in the Brassicaceae suggesting that these genes were lost in the lineage leading to A. thaliana and B. rapa after its separation from the lineage leading to T. hassleriana. Another 132 (subset G) genes are found only in C. papaya and T. hassleriana indicating that these are Brassicales-specific genes that were lost in the Brassicaceae. 453 genes are shared between T. hassleriana and $A$. thaliana but not found in $B$. rapa suggesting that they were lost in the lineage leading to $B$. rapa. Conversely, only 246 genes were lost in the lineage leading to $A$. thaliana and are shared between B. rapa and $T$. hassleriana (subset $\mathrm{C}$ ).

An astonishing number of 5600 contigs (subset Z) could not be matched with high confidence to any other sequence 


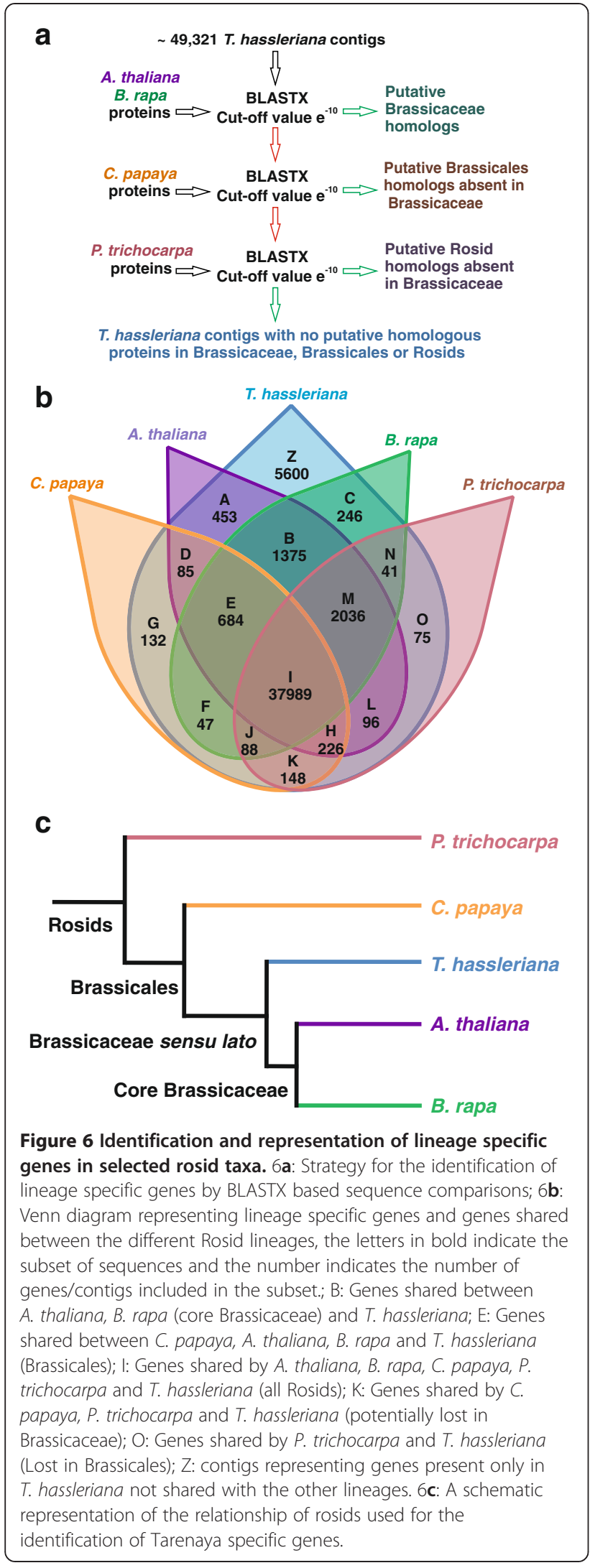

from $P$. trichocarpa, C. papaya, A. thaliana and B. rapa. Of these contigs only 82 could be assigned to 353 GO terms, but a vast majority of the contigs could not be annotated attributing to no significant BLAST hits. A sequence length histogram for these contigs (Additional file 2: Figure S2) shows a bias towards shorter sequences when compared to the sequence length histogram of all contigs (Additional file 2: Figure S1b) suggesting that these were too short for proper annotation and/or may represent 5' and 3' UTR regions of transcripts. Another reason for the small number of annotated genes is because most of the current annotations are based on $A$. thaliana, B. rapa and P. trichocarpa and we already subtracted the sequences orthologous to them. The GO annotations for the T. hassleriana specific genes are the following: cellular process (26.98\%), metabolic process $(29.36 \%)$, response to stimulus (4.76\%), biological regulation $(4.7 \%)$, development $(1.5 \%)$, cell proliferation (1.5\%), reproduction (6.34\%) and signaling processes $(4.76 \%)$ (Additional file 1: Table S6).

\section{Discussion}

In this work we present the floral transcriptome sequence of $T$. hassleriana, which is a member of the Cleomaceae and thus a sister taxon to the Brassicaceae. The transcriptome was analyzed by rarefaction analysis and shown to be of sufficient depth to also identify rare transcripts. As normalization was not carried out, abundance of transcripts could be assessed in silico and compared to qRT-PCR data. The leaf transcriptome of T. hassleriana has been published earlier [10] allowing for comparison of transcript abundance between the leaf and the floral transcriptome. We also attempted to compare the expression of genes represented in the floral transcriptome with the expression of their respective $A$. thaliana orthologs based on presence/absence of expression in the microarray dataset [22] including all flower developmental stages. In addition, we are able to identify 5600 putative transcripts that are specific to the Cleomaceae and 684, which are shared only among the Brassicales C. papaya, T. hassleriana, B. rapa, and A. thaliana.

During assembly, annotation and analysis of the reads obtained by 454 sequencing we observed several challenges. In our study we correlated the in silico floral transcriptome expression in $T$. hassleriana with the conventional qRT-PCR expression of arbitrarily chosen genes with low, moderate, and high expression levels for validation of the transcriptome sequencing expression data. For the in silico expression data we applied two approaches, one was to map the individual reads to the annotated A. thaliana CDS sequences (TSE1), with the advantage that expression data for putative T. hassleriana orthologs of $A$. thaliana genes can be generated without a prior genome sequence information of $T$. hassleriana, thus individual reads are not assembled into contigs. The second 
approach was the de novo assembly of the reads into contigs that are then annotated and the reads are mapped on to these contigs (TSE2). Comparing both methods with qRT-PCR data, the TSE1 approach clearly matches better than the TSE2 approach. One reason for this finding is the presence of chimeric contigs composed of more than one gene, in such cases reads to multiple genes are mapped onto the same contig flaring up the expression. This problem is avoided in TSE1 when the reads are mapped onto orthologous sequences in A. thaliana. Another reason for the disparity between the qRT-PCR expression and TSE2 is due to assembly of contigs with additional non coding nucleotide sequences. This phenomenon was observed in the case of RBCS1A amongst the genes analyzed. The assembled contig was 838 nucleotides long whereas the coding sequence of this gene is $\sim 540$ nucleotides across many plant lineages. The additional 295 nucleotides at the 3' end could represent the 3' UTR nonetheless reads would be mapped to such sequences leading to an overestimation of expression. The third reason for the differences in the expression between qRT-PCR and TSE2 may be the length of the assembled contig versus transcript size, as larger transcripts are fragmented prior cDNA library preparation. For TSE1, qRT-PCR expression data were normalized to A. thaliana CDS lengths and in case of TSE2 to T. hassleriana contig length. The necessity of normalization was seen in case of gene SPL7. The SPL7 contig length was 629 nucleotides whereas the coding sequence of SPL7 in A. thaliana is 2406 nucleotides, thus when the expression is normalized for length of the contig it leads to a much higher expression than when normalized to the length of the A. thaliana ortholog. In case where the contig length matched to the coding sequence length and when the contig had very low or almost no unknown sequences incorporated the qRT-PCR expression matched very well to the TSE2 as was observed in case of TT4.

However, since $T$. hassleriana and A. thaliana have, independent $\alpha$-WGDs, the retention and loss of gene copies following the duplication will be different. By mapping the $T$. hassleriana reads onto the $A$. thaliana orthologs identical sets of orthologous gene copies are assumed for both species leading to over or underestimation of transcript abundance. These illustrated pitfalls for calculating gene expression from RNAseq experiments without the availability of a high-quality reference transcriptome or genome require thorough independent validation of gene expression data.

This work was initiated as a primer to identify genes that may contribute to the morphological differences between the T. hassleriana and the A. thaliana flower. Our focus was mainly on coloration, flowering time, and floral organ size as these are traits that show obvious differences between the two species.
T. hassleriana petals show a deep pink coloration which, when the flower opens, fades into light pink after a few days of exposure to the sun. While in most species only the epidermal petal cell layer is pigmented, T. hassleriana also has pigmented mesophyll cells [14]; suggesting an expansion of the anthocyanin regulation and biosynthesis pathway from petal to mesophyll cells. The pink pigments found in T. hassleriana flowers are acetylated cyanidin diglucoside (sophorosyl)-5-glucosides and acetylated pelargonidin sophorosyl-5-glucosides [14]. All genes required for the synthesis of pelargonidin3 -glucoside and cyanidin-3-glucoside are present in the flower transcriptome. Genes encoding proteins required for the early steps of anthocyanin up to the flavonoid myricetin are also found expressed in the leaf transcriptome, while the genes participating in later steps such as DFR and $L D O X$ are restricted to the flower. These two enzymes are also not expressed in $A$. thaliana flowers but during seed development and late stages of leaf senescence [32].

Transcription factors of the MYB, bHLH, and WD40 families regulate the expression of anthocyanin biosynthesis genes in A. thaliana and Zea mays. While early biosynthesis genes, and their regulators such as AtMYB11, AtMYB12, and AtMYB111 are involved in the production of flavonols, late biosynthesis genes and their regulators are required for the synthesis of anthocyanins from flavonols [37] and references therein. While the putative T. hassleriana orthologs of AtMYB11 and AtMYB12 are hardly expressed in the flower transcriptome, the putative AtMYB111 ortholog shows very strong and flower specific expression suggesting a more prominent role for this gene in the regulation of early biosynthesis genes than for the putative orthologs of AtMYB11 and AtMYB12. Orthologs of the regulators of late anthocyanin biosynthesis in A. thaliana AtTTG1 (WD40 family member), AtTT8, AtGL3, AtEGL3 (all bHLH family members) and AtPAP2 (MYB family member) are also found expressed in the $T$. hassleriana flowers. The T. hassleriana orthologs of A. thaliana genes AtTTG1, AtTT8, AtGL3, AtEGL3, and AtPAP2 forming the late anthocyanin biosynthesis regulatory complex show an approximately similar transcript abundance suggesting that they may function in a complex similar to the one in $A$. thaliana, only with an expression domain expanded to the floral organs.

$A$. thaliana late regulators are mainly expressed in senescing leaves and during seed development (Table 2), but most likely, their expression domain in T. hassleriana has expanded into the flower leading to the pink coloration of the floral organs. A similar situation is found in petunia, where, among others genes AN1, AN11, AN2 and AN4 form complexes similar to that in $A$. thaliana to regulate anthocyanin biosynthesis in the flower [38].

T. hassleriana has, unlike $A$. thaliana, large oval shaped petals, and indeed orthologs of genes involved in limiting 
growth of floral organs were found to be hardly expressed in the T. hassleriana floral transcriptome. BIG BROTHER, encoding for a E3 ubiquitin-ligase represses cell proliferation in all $A$. thaliana proliferating tissues and is expressed strongly and uniformly in all developmental stages of the flower independently of other pathways while being a direct target of the petal organ identity gene AP3 [39-41]. In the T. hassleriana floral transcriptome it has a very low expression of 9 RPKM, suggesting that this may be a reasonable candidate to account for the differences in petal size between the two species.

The particular T. hassleriana hybrid used in this study is sterile even though it produces all the floral organ whorls in the right number and position. However, even though the anthers developed, they did not produce any pollen and also did not dehisce rendering the plants male sterile. While orthologs of $A$. thaliana regulators of anther development were expressed in the the $T$. hassleriana flower, no expression of ROXY1 and ROXY2 was detected. These two genes redundantly control the anther lobe and pollen mother cell differentiation downstream of $S P L$ in A. thaliana [34]. Moreover, only very low expression (6 RPKM) was observed for the T. hassleriana ortholog of DYT1 which acts directly downstream of the $R O X Y$ genes. The phenotype of the T. hassleriana anthers also resembles the roxy1 roxy2 double mutant anther phenotype in A. thaliana, suggesting that our $T$. hassleriana hybrid may lack functional ROXY genes leading to male sterility. We corroborated this observation by qRT-PCR expression data which not only detected very low ROXY1 expression (the only ROXY ortholog in $T$. hassleriana genome [35]) in the mid developmental stage but also showed the de-regulation of expression of the upstream and downstream genes throughout bud development which may provide a cause for the male sterility.

T. hassleriana is perpetually flowering and a sharp transition to flowering as in A. thaliana cannot be observed. Several genes involved in flowering time regulation in A. thaliana are differently regulated in leaves and flowers and we compared the expression of their orthologs in the flower and leaf transcriptomes. FRI is a protein involved in activating transcription via chromatin remodeling of the central floral repressor FLC in A. thaliana [42] and is expressed rather uniformly throughout the plant. However, in T. hassleriana, FRI ortholog expression is not found in leaves. This may suggest a different mechanism for FLC ortholog activation in T. hassleriana leaves, as FLC is expressed there without the presence of FRI.

Interestingly; the expression of two more genes most likely involved in the change from vegetative to reproductive phase in $T$. hassleriana is different from $A$. thaliana. The A. thaliana gene $S M Z$ is expressed in young seedlings, during floral transition and seed maturation [43] unlike its T. hassleriana ortholog which is expressed in flowers and developing buds. Possibly, the T. hassleriana SMZ has function different from its A. thaliana ortholog, which is a rather strong repressor of flowering.

Another candidate gene in the group of flowering time regulators that are differentially regulated in $A$. thaliana and T. hassleriana is ATC. In A. thaliana, ATC is strongly expressed in the root and a small fraction (1-5\%) of its mRNAs moves a long distance to the plant's apex [44]. Notably, we find a significant amount of reads in flower tissue, too many to attribute them to long distance RNA transport. More likely, the $A T C$ homolog is expressed in $T$. hassleriana floral tissue and may be transported throughout the plant to enable the vegetative shoots to first reach sufficient size to start flowering.

\section{Conclusions}

Taken together we conclude from our expression data that a number of floral regulators show expression distinct from that in A. thaliana suggesting that differences in life history traits such as perpetual flowering and pigmentation may be regulated by similar components of regulatory networks in A. thaliana and T. hassleriana that are highly conserved in coding sequence but expressed in a different way in the two species, suggesting that modifications in expression pattern account for a large part of the diversity in flowers and plant life history traits.

\section{Additional files}

\begin{abstract}
Additional file 1: Table S1. 454 sequencing statistics. Table S2. Sequences of the oligonucleotides used for the qRT-PCR. Table S3. List of gene names along with their abbreviations and AGl identifiers. Table S4. P-value calculations using one way ANOVA for analyzing the statistical significance of difference between expression values by qRT-PCR and Transcriptome Sequencing Expression (TSE). Table S5. GO annotation of putatively homologous gene pairs expressed in the T. hassleriana floral transcriptome but not expressed in the A. thaliana floral transcriptome. Table S6. GO annotation of T. hassleriana specific sequences not found in A. thaliana, B. rapa, C. papaya, and P. trichopoda using Blast2GO $\mathrm{G}^{\oplus}$ with BLASTX searches.
\end{abstract}

Additional file 2: Figure S1. 454 sequencing statistics. Figure S2. Read length distribution of $T$. hassleriana lineage specific contigs without any GO annotation. Figure S3. Correlation plot of TSE1 expression by RNA seq and qRT-PCR gene expression.

Additional file 3: T. hassleriana floral transcriptome gene expression.

\section{Abbreviations}

WGD: Whole genome duplication; At-a: A. thaliana alpha WGD; At- $\beta$ : A. thaliana beta WGD; Cq: Quantification cycle; EST: Expressed sequence tag; MYA: Million years ago; RPKM: Reads per kilobase gene model per mappable million; SDR: Standard dose response; Th-a: T. hassleriana alpha WGD; TSE: Transcriptome sequencing expression; TSE1: TSE mapped to A. thaliana CDS sequences; TSE2: TSE mapped to T. hassleriana contigs.

\section{Competing interests}

The authors declare that they have no competing interests.

\section{Authors' contributions}

A. Bhide performed the molecular biology experiments, comparative and in silico expression analysis and drafted the manuscript. SS carried out RNAseq 
assembly, annotation, in silico expression analysis, lineage specific gene discovery. MR performed the rarefaction analysis, A. Becker coordinated and designed the study, and helped to draft the manuscript. A. Becker and APMW conceived the study. All authors helped to improve the manuscript, read, and approved of the final manuscript.

\section{Acknowledgments}

We thank the University of Bremen and the Justus-Liebig University for funding of A. Bhide's position, work in A. Becker's lab is largely funded by the DFG (German Research Foundation). A.P.M.W. acknowledges funding by DFG Priority Program 1529 (Adaptomics). Library preparation and subsequent 454 sequencing was performed by René Deenen at the Biomedical Research Center (BMFZ) of the Heinrich-Heine-University Düsseldorf.

\section{Author details}

${ }^{1}$ Justus-Liebig-Universität Gießen, Institute of Botany, Plant Development Group, Heinrich-Buff-Ring 38, 35392 Gießen, Germany. ${ }^{2}$ Institute of Plant Biochemistry, Cluster of Excellence on Plant Sciences (CEPLAS) Heinrich-Heine-University, Universitätsstr. 1, D-40225 Düsseldorf, Germany. ${ }^{3}$ Department of Biology and Chemistry, University of Bremen, Leobener Str. NW2, D- 28359 Bremen, Germany.

Received: 8 August 2013 Accepted: 6 February 2014

Published: 19 February 2014

\section{References}

1. Itis HH, Cochrane TS: Studies in the Cleomaceae V: a new genus and ten new combinations for the flora of North America. J Bot Nomend 2007, 17:447-451

2. Inda LA, Torrecilla P, Catalán P, Ruiz-Zapata T: Phylogeny of Cleome L. and its close relatives Podandrogyne Ducke and Polanisia Raf. (Cleomoideae, Cleomaceae) based on analysis of nuclear ITS sequences and morphology. Plant Syst Evol 2008, 274:111-126.

3. Hall JC, Sytsma KJ, Iltis HH: Phylogeny of Capparaceae and Brassicaceae based on chloroplast sequence data. Am J Bot 2002, 89:1826-1842.

4. Kers LE: Capparaceae. In Flowering Plants Dicotyledons. Edited by Kubitzki K Bayer C. Berlin, Heidelberg: Springer Berlin Heidelberg; 2003:36-56.

5. Rodman J, Soltis P, Soltis D, Sytsma K, Karol K: Parallel evolution of glucosinolate biosynthesis inferred from congruent nuclear and plastid gene phylogenies. Am J Bot 1998, 85:997.

6. Schranz ME: Independent ancient polyploidy events in the sister families Brassicaceae and Cleomaceae. Plant Cell Online 2006, 18:1152-1165.

7. Couvreur TLP, Franzke A, Al-Shehbaz IA, Bakker FT, Koch MA, Mummenhoff K: Molecular phylogenetics, temporal diversification, and principles of evolution in the mustard family (Brassicaceae). Mol Biol Evol 2009, 27:55-71.

8. Barker MS, Vogel H, Schranz ME: Paleopolyploidy in the Brassicales: analyses of the cleome transcriptome elucidate the history of genome duplications in Arabidopsis and other Brassicales. Genome Biol Evol 2009, 1:391-399.

9. Koteyeva NK, Voznesenskaya EV, Roalson EH, Edwards GE: Diversity in forms of C4 in the genus Cleome (Cleomaceae). Ann Bot 2011, 107:269-283.

10. Brautigam A, Kajala K, Wullenweber J, Sommer M, Gagneul D, Weber KL, Carr KM, Gowik U, Mass J, Lercher MJ, Westhoff P, Hibberd JM, Weber APM: An mRNA blueprint for C4 photosynthesis derived from comparative transcriptomics of closely related C3 and C4 species. Plant Physiol 2011, 155:142-156.

11. Stout AB: Alternation of Sexes and Intermittent Production of Fruit in the Spider Flower (cleome Spinosa). New York: New York Botanical Garden; 1923 [Contributions from the New York Botanical Garden].

12. Patchell MJ, Bolton MC, Mankowski P, Hall JC: Comparative floral development in Cleomaceae reveals two distinct pathways leading to monosymmetry. Int J Plant Sci 2011, 172:352-365.

13. Randall RP: A Global Compendium of Weeds. 2nd edition. 2012

14. Nozzolillo C, Amiguet VT, Bily AC, Harris CS, Saleem A, Andersen, Oyvind M Jordheim M: Novel aspects of the flowers and floral pigmentation of two Cleome species (Cleomaceae), C. hassleriana and C. serrulata. Biochem Syst Ecol 2010, 38:361-369.

15. Chomczynski $P$, Sacchi N: Single-step method of RNA isolation by acid guanidinium thiocyanate-phenol-chloroform extraction. Anal Biochem 1987, 162:156-159.

16. Kent WJ: BLAT--the BLAST-like alignment tool. Genome Res 2002, 12:656-664.
17. Schliesky S, Gowik U, Weber A, Andreas PM, Bräutigam A: RNA-Seq assembly - are we there yet? Front Plant Sci 2012, 3.

18. R Core Team: R: A Language and Environment for Statistical Computing Vienna, Austria; 2013.

19. Bustin SA, Benes V, Garson JA, Hellemans J, Huggett J, Kubista M, Mueller R, Nolan T, Pfaffl MW, Shipley GL, Vandesompele J, Wittwer CT: The MIQE guidelines: minimum information for publication of quantitative real-time PCR experiments. Clin Chem 2009, 55:611-622.

20. Marshall OJ: PerlPrimer: cross-platform, graphical primer design for standard, bisulphite and real-time PCR. Bioinformatics 2004, 20:2471-2472.

21. Livak KJ, Schmittgen TD: Analysis of relative gene expression data using real-time quantitative 5PCR6 and the 2-Itextgreek5DD6CT method. Methods 2001, 25:402-408.

22. Schmid M, Davison TS, Henz SR, Pape UJ, Demar M, Vingron M, Schölkopf B, Weigel D, Lohmann JU: A gene expression map of Arabidopsis thaliana development. Nat Genet 2005, 37:501-506.

23. Conesa A, Gotz S, Garcia-Gomez JM, Terol J, Talon M, Robles M: Blast2GO a universal tool for annotation, visualization and analysis in functional genomics research. Bioinformatics 2005, 21:3674-3676.

24. Tipper JC: Rarefaction and rarefiction; the use and abuse of a method in paleoecology. Paleobiology 1979, 5:423-434

25. Hale MC, McCormick CR, Jackson JR, DeWoody JA: Next-generation pyrosequencing of gonad transcriptomes in the polyploid lake sturgeon (Acipenser fulvescens): the relative merits of normalization and rarefaction in gene discovery. BMC Genomics 2009, 10:203.

26. Lohmann JU, Weigel D: Building beauty: the genetic control of floral patterning. Dev Cell 2002, 2:135-142.

27. Broun P: Transcriptional control of flavonoid biosynthesis: a complex network of conserved regulators involved in multiple aspects of differentiation in Arabidopsis. Curr Opin Plant Biol 2005, 8:272-279.

28. Weiss J, Delgado-Benarroch L, Egea-Cortines M: Genetic control of floral size and proportions. Int J Dev Biol 2005, 49:513-525.

29. Zhang X, Feng B, Zhang Q, Zhang D, Altman N, Ma H: Genome-wide expression profiling and identification of gene activities during early flower development in Arabidopsis. Plant Mol Biol 2005, 58:401-419.

30. Gou J, Felippes FF, Liu C, Weigel D, Wang J: Negative regulation of anthocyanin biosynthesis in Arabidopsis by a miR156-targeted SPL transcription factor. Plant Cell Online 2011, 23:1512-1522.

31. Logacheva MD, Kasianov AS, Vinogradov DV, Samigullin TH, Gelfand MS, Makeev VJ, Penin AA: De novo sequencing and characterization of floral transcriptome in two species of buckwheat (Fagopyrum). BMC Genomics 2011, 12:30.

32. Winter D, Vinegar B, Nahal H, Ammar R, Wilson GV, Provart NJ, Baxter I: An "Electronic fluorescent pictograph" browser for exploring and analyzing large-scale biological data sets. PloS One 2007, 2:e718.

33. Ni W: Regulation of flower development in Arabidopsis by SCF complexes. Plant Physiol 2004, 134:1574-1585.

34. Xing S, Zachgo S: ROXY1 and ROXY2, two Arabidopsis glutaredoxin genes, are required for anther development. Plant J 2008, 53:790-801.

35. Cheng S, van den Bergh E, Zeng P, Zhong X, Xu J, Liu X, Hofberger J, de Bruijn S, Bhide AS, Kuelahoglu C, Bian C, Chen J, Fan G, Kaufmann K, Hall JC, Becker A, Brautigam A, Weber APM, Shi C, Zheng Z, Li W, Lv M, Tao Y, Wang J, Zou H, Quan Z, Hibberd JM, Zhang G, Zhu X, Xu X, et al: The tarenaya hassleriana genome provides insight into reproductive trait and genome evolution of crucifers. Plant Cell 2013, 25:2813-2830.

36. Beilstein MA, Nagalingum NS, Clements MD, Manchester SR, Mathews S: Dated molecular phylogenies indicate a Miocene origin for Arabidopsis thaliana. Proc Natl Acad Sci 2010, 107:18724-18728.

37. Petroni K, Tonelli C: Recent advances on the regulation of anthocyanin synthesis in reproductive organs. Plant Sci 2011, 181:219-229.

38. Quattrocchio F: PH4 of petunia is an R2R3 MYB protein that activates vacuolar acidification through interactions with basic-helix-loop-helix transcription factors of the anthocyanin pathway. Plant Cell Online 2006, 18:1274-1291.

39. Disch S, Anastasiou E, Sharma VK, Laux T, Fletcher JC, Lenhard M: The E3 ubiquitin ligase BIG BROTHER controls Arabidopsis organ size in a dosage-dependent manner. Curr Biol 2006, 16:272-279.

40. Wuest SE, O'Maoileidigh DS, Rae L, Kwasniewska K, Raganelli A, Hanczaryk K, Lohan AJ, Loftus B, Graciet E, Wellmer F: Molecular basis for the specification of floral organs by APETALA3 and PISTILLATA. Proc Natl Acad Sci 2012, 109:13452-13457. 
41. Krizek BA, Anderson JT: Control of flower size. J Exp Bot 2013, 64:1427-1437.

42. Choi K, Kim J, Hwang H, Kim S, Park C, Kim SY, Lee I: The FRIGIDA complex activates transcription of $\mathrm{FLC}$, a strong flowering repressor in Arabidopsis, by recruiting chromatin modification factors. Plant Cell Online 2011, 23:289-303.

43. Mathieu J, Yant LJ, Mürdter F, Küttner F, Schmid M, Dean C: Repression of flowering by the miR172 target SMZ. PLoS Biol 2009, 7:e1000148.

44. Huang N, Jane W, Chen J, Yu T: Arabidopsis thaliana CENTRORADIALIS homologue ( ATC) acts systemically to inhibit floral initiation in Arabidopsis. Plant J 2012, 72:175-184.

doi:10.1186/1471-2164-15-140

Cite this article as: Bhide et al: Analysis of the floral transcriptome of Tarenaya hassleriana (Cleomaceae), a member of the sister group to the Brassicaceae: towards understanding the base of morphological diversity in Brassicales. BMC Genomics 2014 15:140.

\section{Submit your next manuscript to BioMed Central and take full advantage of:}

- Convenient online submission

- Thorough peer review

- No space constraints or color figure charges

- Immediate publication on acceptance

- Inclusion in PubMed, CAS, Scopus and Google Scholar

- Research which is freely available for redistribution 